\title{
Simulation Study of DP-QPSK Coherent Detection Transmission System Based on Optisystem
}

\author{
Yue Yu, Huiwen Yin, Zhiping Huang \\ College of Artificial Intelligence, National University of Defense Technology, Changsha, China \\ Email: huangzhiping65@nudt.edu.cn
}

How to cite this paper: Yu, Y., Yin, H.W. and Huang, Z.P. (2020) Simulation Study of DP-QPSK Coherent Detection Transmission System Based on Optisystem. Optics and Photonics Journal, 10, 134-140. https://doi.org/10.4236/opj.2020.106014

Received: May 26, 2020

Accepted: June 26, 2020

Published: June 29, 2020

\begin{abstract}
Dual polarization quadrature phase shift keying (DP-QPSK) is currently the main modulation format for high-speed optical fiber communications, and its simulation modeling has important implications for theoretical research and practical applications. Based on Optisystem 15 optical communication system simulation software, this paper builds a $100 \mathrm{Gbit} / \mathrm{s}$ rate DP-QPSK modulation format coherent optical transmission system simulation model, analyzes the working principles of the transmitting end, transmission link, and coherent receiver. The principle of signal recovery at the digital signal processing end is briefly explained. The simulation results show that the signal quality after DSP processing has been significantly improved.
\end{abstract}

\section{Keywords}

DP-QPSK, Optisystem 15, Simulation, Coherent

\section{Introduction}

In recent years, with the rapid development of the Internet, user business requirements are constantly changing, data traffic is rising, and new technologies such as big data, cloud computing, large-scale data centers, and the recent rapid development of $5 \mathrm{G}$ are emerging. Latency, high bandwidth and massive data communication have put forward higher requirements on the transmission speed and quality of the backbone network [1].

OTN is an optical transmission technology proposed to overcome the shortcomings of traditional SDH and WDM technologies. The coherent detection technology of OTN signals has been the focus of research in the field of optical fiber communications as the core link of OTN signal reception. The DP-QPSK 
transmission system based on coherent detection has become the industry's mainstream OTN signal transmission scheme with a transmission rate of 100 Gbit/s, and its research has important theoretical and application value [2].

\section{Principle and Structure of DP-QPSKsystem}

\subsection{Transmitting End}

After serial-to-parallel conversion and NRZ encoding, the original signal is divided into two pairs and enters two IQ modulators respectively. The carrier light source passes through a polarization beam splitter (PBS) and is divided into two light waves(X direction and $\mathrm{Y}$ direction) whose polarization directions are mutually perpendicular Two IQ modulators respectively modulate the light waves in these two directions to obtain two QPSK signals with orthogonal polarization, and then pass through the polarization combiner to become a DP-QPSK signal into the transmission link [1].

For the carrier modulation of $\mathrm{X}$ and $\mathrm{Y}$ polarization states, the modulation process is the same. The modulation process of the IQ modulator is analyzed by the carrier modulation of $\mathrm{X}$ polarization state. The structure is shown in Figure 1.

The transfer function of a Mach-Zehnder modulator (MZM) operating in push-pull mode can be expressed as:

$$
E_{\text {out }}=E_{\text {in }}(t) \cdot\left(\frac{\Delta \varphi(t)}{2}\right)=E_{\text {in }}(t) \cdot \cos \left(\frac{u(t)}{2 V_{\pi}}\right) .
$$

The transmission equation in the in-phase shunt is:

$$
E_{X I}=\frac{\sqrt{2}}{2} j E_{X}(j \beta L) \cos \left(\pi k_{X I}\right) .
$$

where $E_{x}$ is the light field intensity input in the $\mathrm{X}$ polarization direction, $\beta$ is the transmission constant, $L$ is the electrode length of MZM, and $k_{X I}=\{0,1\}$.

Similarly, the transmission equation of the orthogonal shunt after passing through the MZM and then through a $90^{\circ}$ phase shifter is:

$$
E_{X Q}=\frac{\sqrt{2}}{2} j E_{X}(j \beta L) j \cos \left(\pi k_{X Q}\right) .
$$

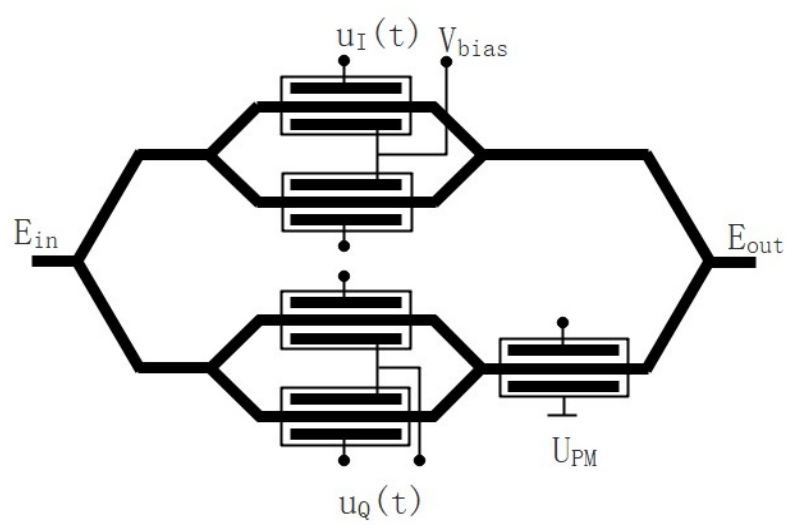

Figure 1. IQ modulator. 
So the transmission equation after the combination of in-phase and quadrature shunt is:

$$
E_{Q P S K, X}=j E_{X}(j \beta L) \exp \left[j \varphi_{X}(t)\right] .
$$

where $\varphi_{X}(t)$ is the modulation phase in the $\mathrm{X}$ polarization direction.

Similarly, the transmission equation of the $\mathrm{Y}$ polarization direction is:

$$
E_{Q P S K, Y}=j E_{Y}(j \beta L) \exp \left[j \varphi_{Y}(t)\right] .
$$

where $\varphi_{Y}(t)$ is the modulation phase in the Y polarization direction.

Finally, the DP-QPSK signal transmission equation after the in-phase and quadrature signals are combined in the polarization combiner can be expressed as:

$$
E_{D P-Q P S K}=\left(\begin{array}{c}
E_{Q P S K, X} \\
E_{Q P S K, Y}
\end{array}\right)=\left(\begin{array}{c}
j E_{X}(j \beta L) \exp \left[j \varphi_{X}(t)\right] \\
j E_{Y}(j \beta L) \exp \left[j \varphi_{Y}(t)\right]
\end{array}\right) .
$$

\subsection{Transmission Link}

Signals in the transmission of optical fiber links are subject to various transmission damages. These damages include fiber loss, spontaneous radiation noise of optical amplifiers, chromatic dispersion, polarization mode dispersion, and nonlinear effects. Distorted waveforms reduce the quality of the signal and seriously affect the transmission performance of fiber optic communication systems [3].

Traditional fiber optic transmission systems often use dispersion-compensating fibers, polarization controllers, and non-chirped fiber Bragg grating devices to compensate and offset these damages. In the coherent detection system, DSP can be used to compensate in the digital domain, which reduces the system cost and greatly improves the flexibility of the system. It can effectively solve the deterioration caused by these damages and improve the coherent detection communication system Transmission performance.

\subsection{Receiving End}

The received optical signal is divided into two $\mathrm{X}$ and $\mathrm{Y}$ optical signals with orthogonal polarization after passing through the polarization beam splitter. The demodulation process for them is the same. The coherent detection process is analyzed with demodulation in X polarization state [4].

The local oscillator light source at the receiving end obtains two $\mathrm{X}$ and $\mathrm{Y}$ signals in the same polarization direction as the received signal after passing through the polarization beam splitter. The $\mathrm{X}$ polarization state local oscillator signal and the received $X$ polarization state optical signal are performed in an 90 ${ }^{\circ}$ mixer Mixing, its structure is shown in Figure 2.

The light signal and the light generated by the local oscillator light source can be expressed as follows:

$$
\begin{aligned}
E_{X} & =\sqrt{P_{X}} \exp \left[j\left(\omega_{X} t+\varphi_{X}\right)\right] . \\
E_{L O} & =\sqrt{P_{L O}} \exp \left[j\left(\omega_{L O} t+\varphi_{L O}\right)\right] .
\end{aligned}
$$




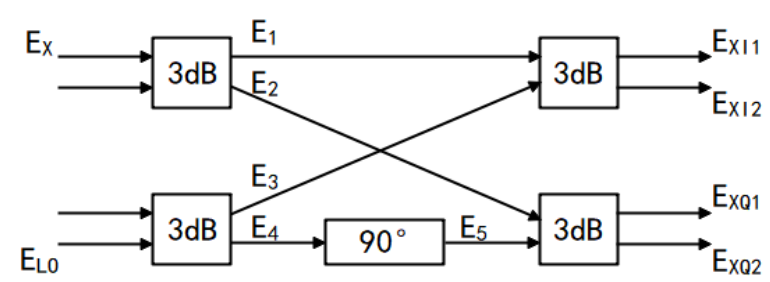

Figure 2. $90^{\circ}$ mixer.

Among them, $P_{X}, \omega_{X}$, and $\varphi_{X}$ are the power, angular frequency, and phase of the received optical signal, and $P_{X L O}, \omega_{X L O}$, and $\varphi_{X L O}$ are the power, angular frequency, and phase of the optical signal of the local oscillator light source, respectively.

The transfer function of the equally divided $3 \mathrm{~dB}$ coupler is:

$$
\left[\begin{array}{c}
E_{\text {out } 1} \\
E_{\text {out } 2}
\end{array}\right]=\frac{\sqrt{2}}{2}\left[\begin{array}{ll}
1 & j \\
j & 1
\end{array}\right]\left[\begin{array}{c}
E_{\text {in } 1} \\
E_{\text {in } 2}
\end{array}\right] \text {. }
$$

Thereby:

$$
\begin{gathered}
{\left[\begin{array}{c}
E_{1} \\
E_{2}
\end{array}\right]=\frac{\sqrt{2}}{2}\left[\begin{array}{cc}
1 & j \\
j & 1
\end{array}\right]\left[\begin{array}{c}
E_{X} \\
0
\end{array}\right]=\frac{\sqrt{2}}{2}\left[\begin{array}{c}
E_{X} \\
j E_{X}
\end{array}\right] .} \\
{\left[\begin{array}{c}
E_{3} \\
E_{4}
\end{array}\right]=\frac{\sqrt{2}}{2}\left[\begin{array}{ll}
1 & j \\
j & 1
\end{array}\right]\left[\begin{array}{c}
0 \\
E_{X L O}
\end{array}\right]=\frac{\sqrt{2}}{2}\left[\begin{array}{c}
j E_{X L O} \\
E_{X L O}
\end{array}\right] .}
\end{gathered}
$$

$E_{4}$ goes through a $90^{\circ}$ phase delay to get $E_{5}=-j E_{4}=-\frac{\sqrt{2}}{2} j E_{X L O}$

Thereby:

$$
\begin{gathered}
{\left[\begin{array}{l}
E_{X I 1} \\
E_{X I 2}
\end{array}\right]=\frac{\sqrt{2}}{2}\left[\begin{array}{ll}
1 & j \\
j & 1
\end{array}\right]\left[\begin{array}{l}
E_{1} \\
E_{3}
\end{array}\right]=\frac{1}{2}\left[\begin{array}{c}
E_{X}-E_{X L O} \\
j E_{X}+E_{X L O}
\end{array}\right] .} \\
{\left[\begin{array}{l}
E_{X Q 1} \\
E_{X Q 2}
\end{array}\right]=\frac{\sqrt{2}}{2}\left[\begin{array}{ll}
1 & j \\
j & 1
\end{array}\right]\left[\begin{array}{l}
E_{2} \\
E_{5}
\end{array}\right]=\frac{1}{2}\left[\begin{array}{c}
j E_{X}+E_{X L O} \\
-E_{X}-j E_{X L O}
\end{array}\right] .}
\end{gathered}
$$

The corresponding powers of the electric fields $E_{X I 1}, E_{X I 2}, E_{X Q 1}$, and $E_{X Q 2}$ are recorded as $P_{X I 1}, P_{X I 2}, P_{X Q 1}$, and $P_{X Q 2}$, respectively. According to the relationship between power and power plant, there are:

$$
\begin{aligned}
P_{X I 1} & =\mathrm{E}\left\{\left[\operatorname{Re}\left\{E_{X I 1}\right\}\right]^{2}\right\} \\
& =\frac{1}{4} P_{X}+\frac{1}{4} P_{X L O}-\frac{1}{2} \sqrt{P_{X} P_{L O}} \cos \left[\left(\omega_{X}-\omega_{X L O}\right) t+\left(\varphi_{X}-\varphi_{X L O}\right)\right] .
\end{aligned}
$$

The current generated by the photodiode corresponding to the balanced receiver is:

$$
I_{X I 1}=\frac{1}{4} R P_{X}+\frac{1}{4} R P_{X L O}-\frac{1}{2} R \sqrt{P_{X} P_{X L O}} \cos \left[\left(\omega_{X}-\omega_{X L O}\right) t+\left(\varphi_{X}-\varphi_{X L O}\right)\right] .
$$

where $R$ is the responsivity of the photodiode. Similarly, we can get:

$$
I_{X I 2}=\frac{1}{4} R P_{X}+\frac{1}{4} R P_{X L O}+\frac{1}{2} R \sqrt{P_{X} P_{X L O}} \cos \left[\left(\omega_{X}-\omega_{X L O}\right) t+\left(\varphi_{X}-\varphi_{X L O}\right)\right] \text {. }
$$




$$
\begin{aligned}
& I_{X Q 1}=\frac{1}{4} R P_{X}+\frac{1}{4} R P_{X L O}-\frac{1}{2} R \sqrt{P_{X} P_{X L O}} \sin \left[\left(\omega_{X}-\omega_{X L O}\right) t+\left(\varphi_{X}-\varphi_{X L O}\right)\right] . \\
& I_{X Q 2}=\frac{1}{4} R P_{X}+\frac{1}{4} R P_{X L O}+\frac{1}{2} R \sqrt{P_{X} P_{X L O}} \sin \left[\left(\omega_{X}-\omega_{X L O}\right) t+\left(\varphi_{X}-\varphi_{X L O}\right)\right] .
\end{aligned}
$$

Bring the above equations into $I_{X I}=I_{X I 2}-I_{X I 1}$ and $I_{X Q}=I_{X Q 2}-I_{X Q 1}$ to get the current output by the $\mathrm{X}$ polarization balanced receiver as:

$$
\begin{aligned}
& I_{X I}(t)=R \sqrt{P_{X} P_{X L O}} \cos \left[\left(\omega_{X}-\omega_{X L O}\right) t+\left(\varphi_{X}-\varphi_{X L O}\right)\right] . \\
& I_{X Q}(t)=R \sqrt{P_{X} P_{X L O}} \sin \left[\left(\omega_{X}-\omega_{X L O}\right) t+\left(\varphi_{X}-\varphi_{X L O}\right)\right] .
\end{aligned}
$$

Similarly, the current output by the $\mathrm{Y}$ polarization balanced receiver is:

$$
\begin{aligned}
& I_{Y I}(t)=R \sqrt{P_{Y} P_{X L O}} \cos \left[\left(\omega_{Y}-\omega_{Y L O}\right) t+\left(\varphi_{Y}-\varphi_{Y L O}\right)\right] . \\
& I_{Y Q}(t)=R \sqrt{P_{Y} P_{X L O}} \sin \left[\left(\omega_{Y}-\omega_{Y L O}\right) t+\left(\varphi_{Y}-\varphi_{Y L O}\right)\right] .
\end{aligned}
$$

It can be seen from the above theoretical derivation that the electrical signal obtained after coherent detection includes the amplitude, frequency and phase information of the received optical signal. After sampling by the ADC, the electrical signal becomes a digital signal and enters the subsequent digital domain for processing [4].

\subsection{Digital Signal Processor}

The digital signal output from the ADC undergoes orthogonalization and normalization processing, and after clock recovery, a series of parameter estimation and compensation algorithms are performed in the DSP. The key algorithms are as follows: dispersion compensation, polarization equalization, frequency offset estimation, carrier phase recovery and so on [1].

\section{Simulation Analysis}

Optisystem is an optical communication system design software developed by Optiwave, with a real device library and a powerful simulation environment. This paper is based on Optisystem 15 and builds a 100 Gbit/s rate DP-QPSK modulation format coherent optical transmission system simulation model using the optical device module built into its software. The structure of the system is shown in Figure 3.

The simulation parameters are set as follows: rate: $100 \mathrm{Gbit} / \mathrm{s}$, light source wavelength: $1550 \mathrm{~nm}$, light source line width: $0.1 \mathrm{MHz}$.

To simulate a real long-distance communication environment, set the fiber length to $100 \mathrm{~km}$, fiber loss to $0.2 \mathrm{~dB} / \mathrm{km}$, amplifier gain to $20 \mathrm{~dB}$, dispersion coefficient to $16.75 \mathrm{ps} / \mathrm{nm} / \mathrm{km}$, the dispersion slope is $0.075 \mathrm{ps} / \mathrm{nm}^{2} / \mathrm{km}$, the polarization film dispersion is $0.5 \mathrm{ps} / \mathrm{km}$, and the cycle number is controlled by the cycle control device to be 2 times, the total length is $200 \mathrm{~km}$, as shown in Figure 4.

Taking the $\mathrm{X}$ branch as an example, the constellation diagram after coherent reception is shown in Figure 5, and it can be seen that the phase information cannot be resolved because of damage on the transmission link. 
Transmitting End

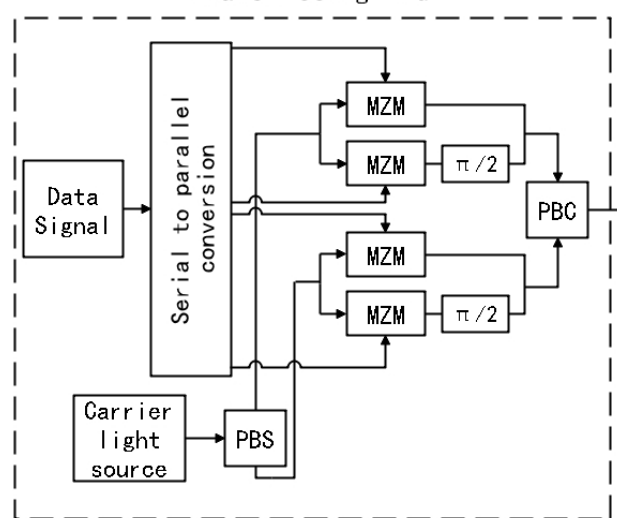

Figure 3. The structure of the system.
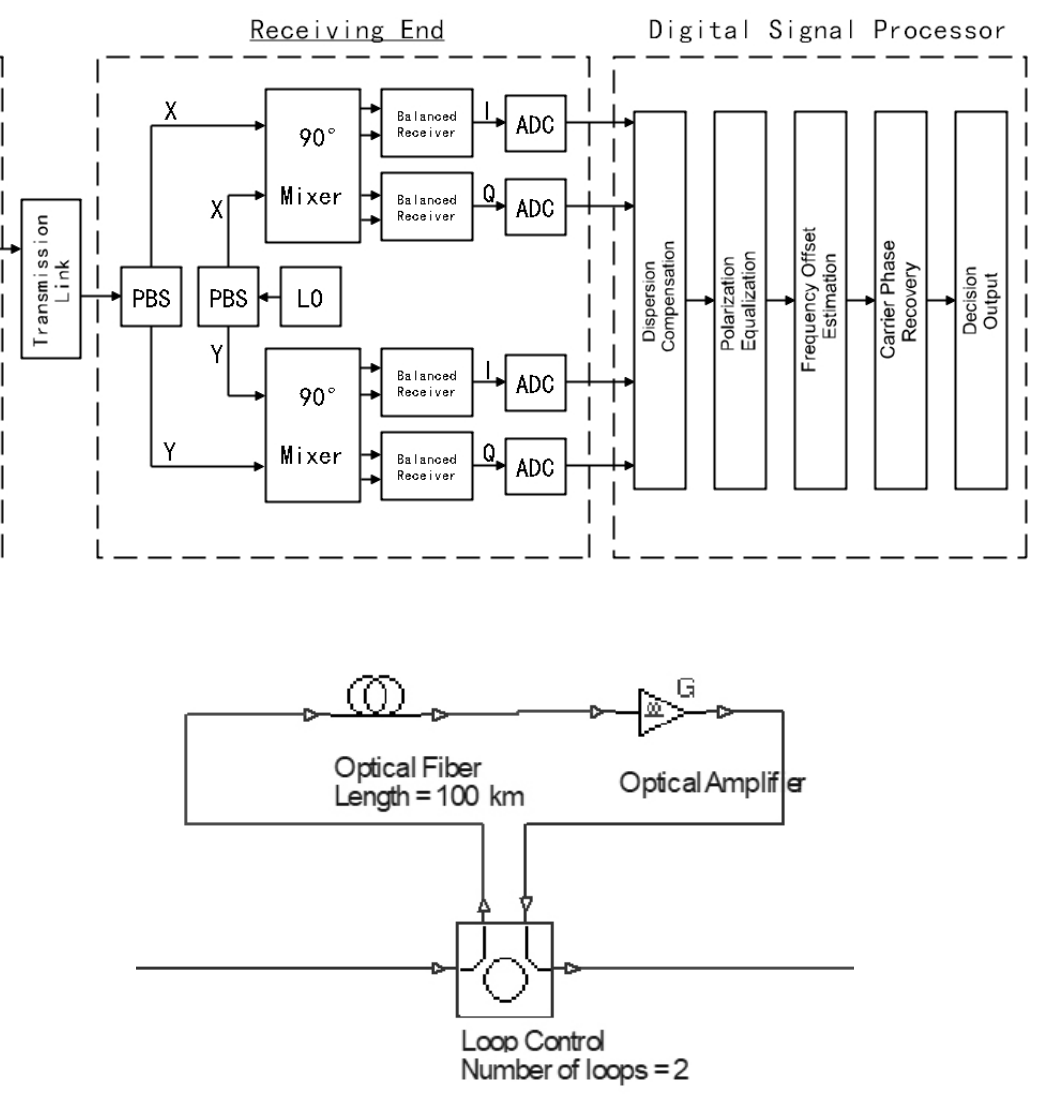

Figure 4. Transmission link.

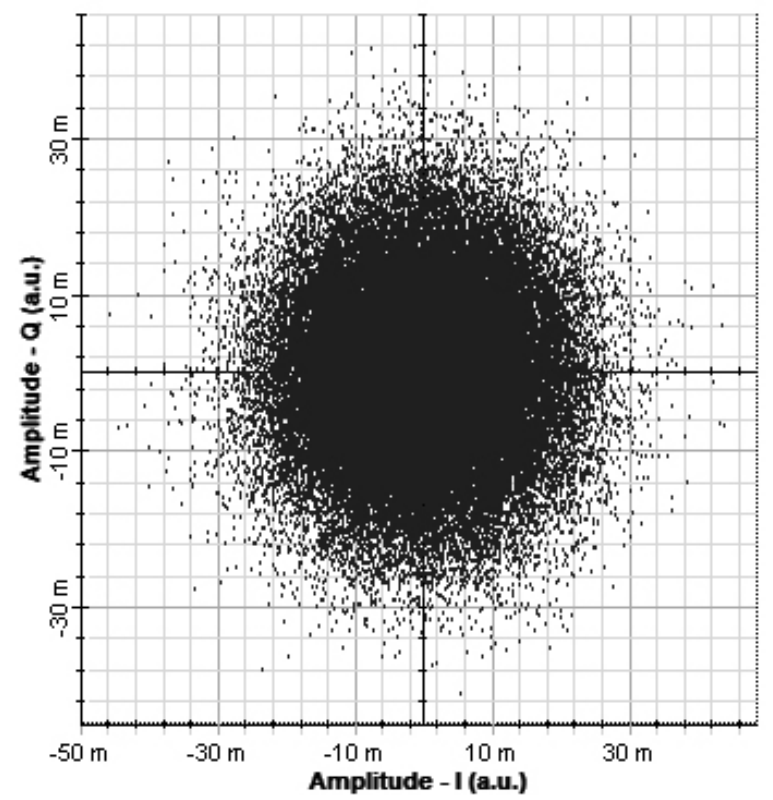

Figure 5. The constellation of $\mathrm{X}$ branch.

After performing a series of compensations through digital signal processing, the resulting constellation is shown in Figure 6. 


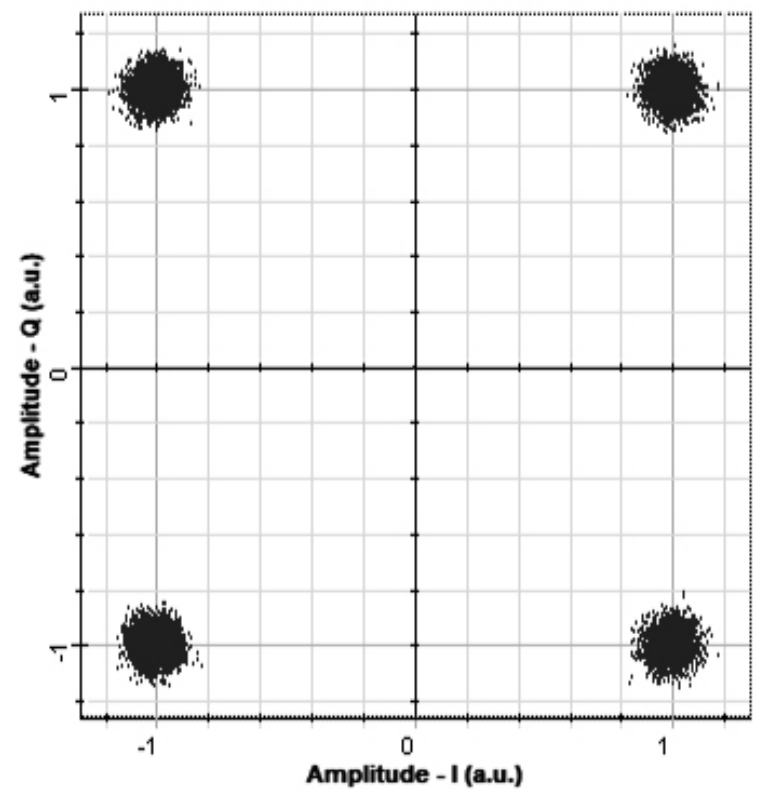

Figure 6. The constellation of X branch after DSP.

\section{Conclusion}

In this paper, the principle of the DP-QPSK modulation format transmission system is analyzed in depth. On this basis, a simulation system is built based on Optisystem 15 software. The simulation results show that after digital signal processing, various damages and phases on the line can be effectively compensated. The information is restored and the transmission performance of the system is significantly improved.

\section{Conflicts of Interest}

The authors declare no conflicts of interest regarding the publication of this paper.

\section{References}

[1] Yu, J.J., Chi, N. and Chen, L. (2013) Coherent Optical Communication Technology Based on Digital Signal Processing. The People's Posts and Telecommunications Press, Beijing.

[2] Kikuchi, K. (2016) Coherent Optical Communication Technology. 201621 st Opto Electronics and Communications Conference (OECC) Held Jointly with 2016 International Conference on Photonics in Switching (PS), Niigata, Japan, 3-7 July 2016, 16424767.

[3] Kuschnemv, M. (2009) DSP for Coherent Single-Carrier Receivers. Lightwave Technol, 27, 3614-3622. https://doi.org/10.1109/JLT.2009.2024963

[4] Kang, J. (2014) The Study of Technology of 100 Gbps DP-QPSK Optical Communication System. MA.Sc Thesis, Beijing Jiaotong University, Beijing. 